\title{
Líneas metodológicas de evaluaciones de impacto ambiental
}

Una evaluación de impacto debe abarcar los siguientes aspectos: describir la acción propuesta, así como otras alternativas; predecir la naturaleza y magnitud de los 'efectos ambientales'; predecir los aspectos humanos; interpretar los resultados y prevenir los efectos ambientales. Además, hay que disponer de una metodología para las fases de comunicación (información al público y al ejecutivo) y, de acuerdo al caso, para los procedimi-entos de inspección durante la fase de construcción y de operación del proyecto o la acción de que se trate.

Palabras clave: Impacto Ambiental; Evaluación; Metodologías.

\section{Linhas metodológicas de avaliações de impacto ambiental}

Uma avaliação de impacto deve abranger os seguintes aspectos: descrever a ação proposta, bem como outras alternativas; prever a natureza e magnitude dos 'efeitos ambientais'; prever aspectos humanos; interpretar os resultados e prevenir os efeitos ambientais. Além disso, deve estar disponível uma metodologia para as fases de comunicação (informação ao público e ao executivo) e, conforme o caso, para os procedimentos de fiscaliza-ção durante a fase de construção e operação do empreendimento ou ação em causa.

Palavras-chave: Impacto Ambiental; Avaliação; Metodologias.

Topic: Notas Científicas

Reviewed anonymously in the process of blind peer.
Received: 11/11/2020

Approved: 15/02/2021
Cleber Vinicius Vitorio da Silva (iD)

Universidade Federal Rural do Rio de Janeiro, Brasil

http://lattes.cnpq.br/4275890458575782

http://orcid.org/0000-0001-8337-9615

clebervitorio88@gmail.com

Pauli Adriano de Almada Garcia

Universidade Federal Fluminense, Brasil

http://lattes.cnpq.br/3866888351512590

http://orcid.org/0000-0002-3934-4332

pauliadriano@gmail.com

Vanessa da Silva Garcia

Universidade Federal Fluminense, Brasil

http://lattes.cnpq.br/2206038698250045

vanessa.sgarcia@gmail.com

\author{
Lais Alencar de Aguiar (iD \\ Universidade Federal do Rio de Janeiro, Brasil \\ http://lattes.cnpq.br/5785500333245448 \\ http://orcid.org/0000-0002-1551-4085 \\ aguiar.lais@gmail.com \\ Josimar Ribeiro de Almeida \\ Universidade do Estado do Rio de Janeiro, Brasil \\ http://lattes.cnpq.br/3215586187698472 \\ http://orcid.org/0000-0001-5993-0665 \\ almeida@poli.ufri.br
}

\section{Referencing this:}

SILVA, C. V. V.; GARCIA, P. A. A.; GARCIA, V. S.; AGUIAR, L. A.; ALMEIDA, J. R.. Líneas metodológicas de evaluaciones de impacto ambiental. Natural Resources, v.11, n.1, p.124-133, 2021. DOI: http://doi.org/10.6008/CBPC2237-9290.2021.001.0015 


\section{INTRODUCCIÓN}

El interés masivo por los temas ambientales surgió al final de los años sesenta. Fue entonces cuando se empezaron a percibir con nitidez los problemas de deterioro del medio ambiente, que en principio se centraron especialmente en la contaminación. El concepto medio ambiente tuvo e nesa época dos acepciones bastante claras, según lo que se aplicara en los países industrializados o en los países en vías de desarrollo. En los primeros, la temática ambiental se concentraba casi exclusivamente en los aspectos de contaminación, en cuyo caso resultaba correcto considerar que los problemas ambientales tenían un carácter tecnológico. En cambio, en los países en vías de desarrollo el concepto era mucho más amplio y de carácter socioeconómico y político, más que tecnológico, puesto que se consideraban problemas ambientales prioritarios, precisamente los derivados del subdesarrollo: problemas sanitarios, condiciones de los asentamientos humanos, falta de viviendas y escuelas, deficiencias de nutrición, de destrucción de bosques y pérdidas de suelos, destrucción o mala explotación de recursos naturales y otros. A estos problemas del subdesarrollo hay que añadir los que pueden generarse de un desarrollo que no considere en sus proyectos la variable ambiental. Hoy en día, en todos los países, el concepto de medio ambiente tiene un sentido único y generalizado, cada vez más amplio, hasta el punto de que conceptos tan complejos y extensos como los de la calidad de vida y asentamientos humanos se integran en su temática. Persiste sin embargo la idea de que los problemas ambientales en los países industrializados derivan en su mayor parte de procesos de desarrollo y, muy especialmente, de las grandes concentraciones humanas y/o industriales (la contaminación, la deshumanización de la vida en las grandes ciudades, la creciente conflictividad social de las metrópolis, el consumo exagerado de recursos naturales y de energía, los problemas urbanísticos, etc.), en cuanto que en los países en vías de desarrollo los problemas ambientales se deben, principalmente, al bajo índice de crecimiento. Las evaluaciones de impacto ambiental nacieron en los Estados Unidos, como consecuencia de la Ley Nacional de Política Ambiental (National Environmental Policy Act- NEPA). Es en ese país donde se han hecho más trabajos de este tipo y, por consiguiente, donde se han desarrollado más metodologías para los mismos. Hasta la fecha se han preparado muchas, pero ninguna de ellas tiene una dimensión o un carácter universal. Así pues, aun que existe gran cantidad de modelos, son pocos los que están sistematizados.

\section{RELATO}

Se dice que hay un impacto ambiental cuando una acción o actividad produce una alteración en el medio o en alguno de los componentes del medio. Por lo tanto, la variable fundamental en estos estudios es la cuantificación de la alteración. En los estudios de impacto ambiental se trata de evaluar las consecuenciasde una acción, para ver la calidad del ambiente que habría con o sin dicha acción. Tales evaluaciones deben realizarse en la fase previa al proyecto, antes que éste se realice, con objeto de: a) efectuar una mejor planificación y formulación de propuestas, desde el punto de vista ambiental y b) considerar adecuadamente los factores ambientales, por parte de las autoridades públicas, cuando aprueben una propuesta o determinen una alternativa. Se aplica el concepto de evaluación del impacto ambiental a un 
estudio encaminado a identificar e interpretar, así como a prevenir, las consecuencias o los efectos que acciones o proyectos determinados pueden causar a la salud y al bienestar humanos y al entorno, o sea, en los ecosistemas enque el hombre vive y de los que depende (ALMEIDA et al., 2019). La dificultad para demostrar la complejidad de la dinámica ambiental en una única definición hace que, en general, todas las definiciones adquieran un carácter reduccionista y estático. Esto constituye, de cierto modo, el gran problema de las conceptualizaciones. Otras dificultades encontradas en la definición y, especialmente, en la identificación de un impacto ambiental consisten en la propia delimitación del impacto, una vez que el mismo se propaga espacial y temporalmente através de una compleja red de interrelaciones y, también, en las deficiencias instrumentales y metodológicas para prever las respuestas de los ecosistemas a las actividades antrópicas. Esta cuestión es todavía más crítica cuando se trata de la dimensión social.

\section{Método}

Es importante resaltar que la investigación guiada por el método dialéctico revela la historicidad del fenómeno y sus relaciones, a un nivel más amplio, sitúan el problema dentro de un contexto complejo, al mismo tiempo, estableciendo y señalando las posibles contradicciones entre los fenómenos investigados. La investigación cualitativa se basa en la inseparabilidad de los fenómenos y su contexto, ya que las opiniones, percepciones y significados se entenderán con mayor profundidad desde el contexto. La validez se referiría a la similitud entre el concepto y sus medidas, el grado en que una medida representa precisamente lo que se espera. La garantía de validez comenzaría con una comprensión directa de lo que se debe medir, por lo tanto, se trata de formulación de la investigación. El método propuesto se dividió en tres fases, que se subdividieron en etapas para la realización del relevamiento bibliográfico. En la primera fase de la investigación (preguntas de investigación), se verifican las principales decisiones y definiciones sobre la investigación. Las preguntas de investigación se destacan y sirven como impulso para el inicio de la revisión. Se define el tema para definir la encuesta bibliográfica y el período disponible. Otras decisiones se toman simultáneamente, como definir palabras clave, para que actúen como identificadores. Paralelamente, resumen los principales temas sobre el tema a investigar; así como combinaciones de palabras clave. Estos, a su vez, se pueden realizar mediante operadores booleanos y en diferentes bases de datos como CAPES Journal Portal, SCIELO Database, Publish or Perish, EBSCO, entre otras. En el presente caso, las bases de datos fueron Web of Science (o ISI), Plataforma SUSTENERE, Scielo y Scopus. Estas bases de datos son de fácil acceso, lo que permite, a través de sus herramientas, realizar una cuidadosa investigación, abarcando un amplio número de revistas. La segunda fase (selección de artículos) inicia la encuesta y selección de artículos, utilizando los criterios de exclusión. Las palabras clave de exclusión se utilizan para realizar una clasificación más cuidadosa de los artículos. También delimitando el período. En el siguiente paso, se produce el primer filtro de los artículos, se realiza una selección de los títulos, identificando los no alineados. En el siguiente paso, se leen los resúmenes para excluir a aquellos que son impertinentes con el tema investigado. El siguiente paso contiene un análisis subjetivo por parte del investigador para identificar, en los artículos 
alineados con la temática, aquellos que tienen mayor relevancia académica. Un análisis bibliométrico indica la relevancia del autor / artículo para la composición de la referencia bibliográfica.

La tercera fase corresponde a la clasificación de los artículos seleccionados. Se organizan muestras de artículos seleccionados. El objetivo es el orden, patrón que variará según las necesidades del investigador y lo priorice como foco de desarrollo temático. Destaca la importancia de un análisis exhaustivo de los artículos que componen la muestra. En la investigación cualitativa, el concepto de validez toma diferentes formas, ya que la discusión de las escalas de medición no se aplica a los métodos cualitativos, lo que requiere una comprensión de la validez desde otra perspectiva. Un atributo que se relaciona con la objetividad, la posibilidad de repetir el experimento, el hecho de que la investigación esté abierta a la verificación de otras personas y la capacidad de generalizar (SOUZA et al., 2011). La validez puede verse genéricamente como la correspondencia entre investigación y realidad (LINS et al., 2015). Se refiere a verificar los resultados como verdaderos y confiables. Estaría relacionado con el hecho de que los resultados reflejan fielmente la situación analizada y son confiables, en el sentido de que no habría razón para dudar de ellos; es decir, la investigación es válida si la evidencia proporciona el soporte necesario para sus conclusiones (ALMEIDA et al., 2019). La intención no es generalizar, sino describir, analizar, buscar comprender.

\section{DISCUSIÓN}

\section{Métodos de Identificación}

\section{Metodología 'Ad Hoc'}

Los métodos 'Ad Hoc', como la propia denominación indica, son basados en el conocimiento empírico de expertos en el asunto y/o del área en cuestión y son elaborados para un proyecto específico. Éstos identifican los impactos mediante un 'brainstorming', los caracterizan y los organizan en listas o matrices. Estas metodologías, utilizadas aisladamente, deberán desarrollar la evaluación de impactos ambientales de forma sencilla, objetiva y de manera disertativa. Son adecuadas para casos con escasez de datos, dando orientación para otras evaluaciones. La ventaja es que se trata de una estimativa rápida de evaluación de impactos de forma organizada, fácilmente comprensible por el público. Sin embargo, no realizan un examen más detallado de las intervenciones y variables ambientales incluidas en el proceso, siendo evaluaciones bastante subjetivas que privilegian los aspectos cualitativos sobre los cuantitativos.

\section{Técnica Delphi}

La técnica Delphi (o Delfos) es, básicamente, un sistema cuyo objetivo esintentar reducir el margen de errores en una toma de decisiones. Se efectúa a mediante una discusión, por parte de un grupo de especialistas, tratando de llegar a un consenso sobre una determinada cuestión. El nombre Delphi viene del lugar de la Grecia antigua, hoy Castri, donde oráculos se reunían para discutir y llegar a decisiones. El uso de esta técnica fue iniciado como un ejercicio de planeamiento estratégico por la Fuerza Aérea de los EUA en 1953. El objetivo del estudio original fue el de "obtener el consenso de opinión más seguro de un grupo de 
expertos utilizando una serie de cuestionarios, intercalados con retroalimentación controlada de opiniones. Sin embargo, por causa del tema de este primer estudio valioso, de la técnica Delphi, fue necesario, más tarde, llamar la atención de individuos fuera del amparo de la comunidad. El trabajo se divulgó bajo el título 'Informe sobre el estudiode la prevención de amplio rango', por T. J. Gordon y Olaf Helmer, publicado como un artículo de Rand en 1964. El estudio se hizo para explorar tanto los aspectos metodológicos de la técnica como para obtener resultados substanciales. Los autores encontraron dentro de la misma tanto un vacuo contiguo como técnicas evaluadas de la prevención de ampliorango. El estudio cubrió seis tópicos: penetración científica, control poblacional, automación, progreso espacial, prevenciónc ontra guerras y sistemas armamentistas, siendo crecientemente empleada en muchos campos de la actividad humana. Hace una buena revisión de la técnica Delphi. En la evaluación ecológica de impactos ambientales, ejemplifica bien el uso de la técnica Delphi. La técnica Delphi está basada en las siguientes premisas. Las opiniones de los especialistas son justificadas como 'inputs' en la toma de decisiones, cuando las respuestas y cuestiones son desconocidas; - El consenso entre un grupo de especialistas permite obtener una respuesta mejor que la de un único especialista. Para una toma de decisión, através de la técnica Delphi, debemos constituir, por lo menos, tres grupos de individuos - Los que toman decisiones - aquéllos que recibirán los resultados deltrabajo de los especialistas y los utilizarán; - Los que elaborarán los cuestionarios que serán enviados por correo a los especialistas y que, después de recibirlos de vuelta, los analizarán; - El grupo de especialistas que deberá responder las cuestiones y remitirlas respuestas por correo. Los participantes del tercer grupo reciben las cuestiones y dan respuestas a las mismas. Esas respuestas serán entonces, listadas, analizadas y los resultados obtenidos pasan a los especialistas del segundo grupo. Cada uno de ellos irá a analizar las informaciones y agruparlas en conjuntos de respuestas semejantes. Vamos a suponer que se llegue a cuatro alternativas. Éstas son, entonces, nuevamente enviadas al grupo 3 para evaluación y selección. Las respuestas vuelven al grupo 2. Repitiendo ese procedimiento se procurará llegar a un consenso único entre todos los miembros del grupo 3. Es extremadamente importante mantener el anonimato entre los especialistas participantes del proceso, especialmente en el grupo B.

¿Cuál es el número de especialistas que deben participar de la discusión? Diez sería probablemente el número ideal, pero ese número puede depender de varios factores, como: disponibilidad de especialistas, gravedad de la cuestión y amplitud de la cuestión tratada. La constitución del grupo 3 puede ser hecha a través de una consulta inicial, por parte del grupo 2, a dos o tres especialistas de reconocida competencia que, a su vez, podrían indicar técnicos a ser contactados para participar de la discusión. Una alternativa de la técnica Delphi es la del grupo nominal que, en vez de utilizar consultas a isladas por correspondencia, reúne a todos los técnicos en un ejercicio cara a cara. A la vez permite discusiones más amplias, incluso con mayor rapidez. Esa técnica presenta algunas desventajas, tales como: cuestiones económicas (traslados, hotel), de tiempo, disponibilidad de los participantes, influencia de cuestiones personales (rivalidades, personalidades) y hasta de naturaleza política. Es indispensable que todos estén bastante motivados, nunca forzados a participar de la discusión. Para organizar un trabajo dentro de la técnica Delphi, debemos hacer lo siguiente: - Seleccionar un grupo de participantes; - Enviar, a cada uno, una carta de invitación explicando las 
finalidades del ejercicio y dando instrucciones sobre el cuestionario al que los participantes deberán responder; - Mandar el formulario con las cuestiones formuladas de forma bastante clara. Las respuestas deben ser analizadas estadísticamente y enviadas a los participantes. Con base en ese análisis, los participantes responden nuevamente las preguntas, hasta llegar al máximo consenso posible entre los mismos. El uso de la técnica Delphi fracasó algunas veces debido, especialmente, a errores como los siguientes: - Imposición de puntos de vista de los técnicos del grupo 2, juicio previo sobre una cuestión; Consideración de que la técnica Delphi puede ser usada en todas las situaciones; - Técnicas insuficientes de resumen y presentación de las respuestas delos grupos; - No saber administrar (o la falta de percepción) las discordancias dentro delos grupos, lo que puede llevar a falsos consensos. La cuestión sobre la organización del grupo debe ser relevante. El mismo debe ser constituido por personas efectivamente capacitadas, sin preocupaciones personales, políticas y/o económicas. Una variante del uso de la técnica Delphi es el llamado Delphi político. Los grupos son, en este proceso, formados por cerca de diez personas que no son técnicos, pero que buscarán evaluar los impactos, las consecuencias de una determinada acción y su aceptabilidad. Se trata de un medio de análisis de políticas y no un mecanismo de toma de decisiones. No tiene el objetivo de obtener un consenso, sino de conocer y evaluar desacuerdos sobre determinadas cuestiones, en especial, sobre su relevancia.

\section{Listas de Chequeo ('Check-List')}

Las listas de chequeo constituyeron el primer método usado en los estudios de impactos ambientales (EIA) y son muy útiles, especialmente, como un estudio preliminar para la identificación de impactos relevantes. Consiste en listas estandarizadas de factores ambientales a partir de las cuales se identifican los impactos provocados por proyectos específicos. Aun así, no permiten proyecciones ni la identificación de impactos secundarios. Existen, actualmente, diversas listas estandarizadas por tipo de proyecto (hídricos, energéticos, de rutas, otros), listas computarizadas como el Programa MERES del Departamento de Energía de los Estados Unidos que, a partir de especificaciones del tamaño y naturaleza de la planta, calcula la emisión de contaminantes en el aire. Los "check-lists" son métodos, básicamente, de identificación cualitativa que pueden también incorporar escalas de valoración y ponderación de factores. Ellos constituyen una forma concisa y organizada de relacionar los impactos, a pesar de ser por demás sencillos y estáticos, no evidenciando las interrelaciones entre los factores ambientales. Hay varios tipos de listas: A) Listas simples: se analizan factores o parámetros sin valorarlos niinterpretarlos. B) Listas descriptivas: se analizan factores o parámetros y se presenta lainformación referida de los efectos sobre el medio ambiente. C) Listas de verificación y escala: se lleva a cabo el mismo trabajo que con la lista descriptiva y se incluye, además, una escala subjetiva de valoración de los efectos ambientales. D) Listas de verificación, escala y ponderación: se opera con el procedimiento de las listas de verificación y escala, pero se introducen unas relaciones de ponderación de factores en las escalas de valoración.

Listas Descriptivas son listas de parámetros ambientales, de fuentes de información y de técnicas de previsión. Listas comparativas buscan comparar los efectos acarreados por las diversas alternativas de un 
proyecto. Consiste en una relación de factores o recursos ambientales con criterios de relevancia para discernir, tanto los valores normales deseables de cada factor o recursos, como su dimensión temporal y, además, identificar alguna indicación de si el proyecto provoca efectos negativos o no sobre el recurso.

Listas en cuestionario consiste en una serie de preguntas procurando abordar los aspectos deposibles impactos por un proyecto. Listas de control de condiciones: en éstas, las acciones son presentadas en columnas y las consecuencias en filas. Cada acción tendrá un peso, que podrá variar de 1 a 5, de acuerdo con su importancia, en relación a los objetivos del análisis. Por otro lado, las consecuencias tendrán un valor por notas, que pueden variar de $-5 a+5$, siendo que: -5 es el valor para el impacto negativo más intenso; -1 es el valor para el impacto negativo menos intenso; 0 (cero) será dado cuando no haya impacto; +1 será el impacto positivo menos intenso; +5 será el impacto más intenso. El peso de cada acción y la nota de cada consecuencia, por ejemplo, en determinada zona de un estuario, serán justificados en el texto y resultará de un análisis crítico, realizado por el equipo, a partir de datos disponibles. Después de la multiplicación del peso por la nota de cada ítem, será obtenido un valor positivo o negativo. De la sumatoria de estos valores, dividida por la suma de los pesos, se obtendrá, para cada zona del estuario, en números finales, en el intervalo (-5 a $+5)$, que será el índice del impacto existente en el estuario. Si el trabajo es desarrollado para varias áreas de un estuario, será posible establecer la jerarquía de las mismas por los índices de impactos obtenidos.

Listas de control escalar. En el caso de existir propuestas alternativas al proyecto o maneras alternativas de implementarlo, se recomienda que la lista de control sea formulada con el objetivo de permitir comparaciones en esas alternativas, con base en alguna unidad escalar seleccionada. Tales listas son también indicadas para la comparación entre la situación anterior al establecimiento del proyecto (o adopción de tecnología) y la situación esperada después de su implantación (o adopción).

Listas de referencias son listas preparadas por técnicos especialistas de diferentes áreas que sirven de referencia cuando se buscan las posibles causas y/o efectos de un impacto ambiental. Ejemplos:

I. Listas de factores correspondientes a impactos geobiofísicos: A. Contaminación atmosférica: partículas sólidas; gases; vapores, aerosoles; sustancias tóxicas; alteración del microclima; B. Contaminación del agua (aguas continentales superficiales, subterráneas y marítimas). Factores de cantidad: caudal y variación de flujo del agua; C. Factores de la calidad del agua: - Físicos: temperatura; turbidez; densidad; sólidos disueltos y en suspensión; color, olor y sabor; - Químicos inorgánicos: oxígeno; hidrógeno; nitrógeno; fósforo; metales alcalino terrosos; azufre; halógenos; carbono inorgánico, silicio; metales pesados ;Químicos orgánicos; - Biodegradables: hidratos de carbono, grasas y proteínas; -No biodegradables: pesticidas, detergentes, hidrocarbonatos y productos petroquímicos persistentes; - Biológicos: organismos patógenos; organismos eutrofizantes; DBO; otros.; D. Sustancias radiactivas; E. Ruido; F. Recursos naturales: vegetación natural; exploración vegetal; uso del suelo agrícola y pastoril; recursos minerales; espacios destinados a usos recreativos; paisaje; medio acuático; recursos pesqueros; G. Factores biológicos: inventario de biotipos y biocenosis y sus correlaciones; fauna: inventario de especies características; flora; inventario de especies características y vegetación natural; especies en peligro de extinción; diversidad de especies; 
estabilidad del ecosistema; H. Suelo: precipitación; deposición; sedimentación; contaminación por residuos sólidos, líquidos o gaseosos; alteración del revestimiento vegetal; otros.

II- Lista de factores correspondientes al impacto socio-económico: A. Área: uso inadecuado del área y de los recursos naturales; modificación en el uso del área; alternativas de uso para el área y los recursos naturales; expropiación de terrenos; B. Alteración del paisaje: destrucción o alteración del paisaje; destrucción de sistemas naturales; C. Aspectos humanos y socioculturales: patrones culturales; destrucción o alteración de la calidad de vida en términos de aspectos culturales e/ohistóricos; enfermedades, consecuencia de congestionamiento urbano y de tráfico; alteración de los sistemas o estilos de vida; tendencia de variación demográfica; lugares históricos que pueden ser afectados; D. Aspectos económicos: estabilidad económica regional; renta y gastos para el sector público; consumo y renta per cápita; empleos que pueden ser producidos en la fase de construcción del proyecto; empleos fijos durante el funcionamiento del proyecto; desarrollo económico de actividades comerciales; servicios, durante la ejecución y funcionamiento del proyecto; viviendas; infraestructura de transportes; infraestructura sanitaria; servicios comunitarios y equipamientos urbanos; otros.

Lista de utilidad para multiatributos: proyectos en los cuales se presentan diversas alternativas generalmente revelan diferentes impactos entre ellos, así como niveles variables de esos impactos. La dificultad consiste en ponderar esa variabilidad en las evaluaciones. Ese método está basado en la teoría de utilidad para multiatributos, en la cual son ponderados los componentes principales en consideración de acuerdo con la definición de su importancia para el proceso estudiado. Aun confiando en la percepción de especialistas, ese método ofrece una sistemática lógica para comparación de impactos y de proyectos. El mismo alcanza independencia ambiental entre parámetros aun que éstos normalmente sean independientes. El método de multiatributos consiste en seleccionar una serie de parámetros indicadores que puedan ser medidos os estimados y que permitan formar un cuadro claro de probabilidades de los impactos. Para cada parámetro se debe obtener medidas del estado, o sea, valores de ocurrencia real y potenciales del parámetro. Para estimarse la ocurrencia potencial con la implantación del proyecto o tecnología, se proyectan los valores a través de modelos y datos de la literatura, o con simulaciones de variados niveles de complejidad (ANDRADE et al., 2014).

\section{CONSIDERACIONES FINALES}

Las líneas metodológicas de evaluación son mecanismos estructurados para comparar, organizar y analizar informaciones sobre impactos ambientales de una propuesta, incluyendo los medios de presentación escrita y visual de esas informaciones. Los métodos para evaluar el impacto ambiental son muy diferentes en un caso u otro. La magnitud del impacto puede deducirse, de un estudio generaly breve de un proyecto, en una evaluación preliminar que debe hacerse siempre, puesto que se opera por aproximaciones sucesivas. Normalmente, son denominados de técnicas os métodos de evaluación los instrumentos que tienen por objetivo identificar, caracterizar y sumariar los impactos de un determinado proyecto o programa. Además de eso, se utilizan en la fase de anteproyecto otras técnicas que corresponden a las diferentes 
disciplinas involucradas en el proceso de evaluación del mismo. Existe a disposición de los evaluadores de impacto del ambiente una gran variedad metodológica, con más de cien métodos descriptos para los más distintas propósitos y situaciones (ALMEIDA et al., 2013). Esa variedad es previsible dada la cantidad de situaciones a ser sometidas a las evaluaciones y a las distintas escalas de calidad y disponibilidad de datos. Debido a la gran diversidad de métodos de evaluación de impactos ambientales existentes, donde muchos no son compatibles con nuestras condiciones socioeconómicas y políticas, se hace necesario seleccionarlos de acuerdo con nuestras propias condiciones y muchas veces hasta adaptarlos, por medio de modificaciones y/o revisiones, para que sean realmente útiles en la toma de decisión de un proyecto. Queda, entonces, a criterio de cada equipo técnico, la selección de aquél (los) método (s) más apropiado (s), o parte (s) de estos, de acuerdo con las actividades propuestas. Así, definir la metodología de evaluación de impactos ambientales consiste en definir los procedimientos lógicos, técnicos y operacionales capaces de permitir que el proceso, antes referido, sea completado. Existen en la literatura diversas clasificaciones para estas técnicas o métodosque varían conforme la óptica adoptada. Existe una división en dos grandes grupos. Por un lado, encontramos los métodos tradicionales de evaluación de proyectos como el análisis costo-beneficio y, por otro lado, métodos basados en el establecimiento de un sistema de escalas valorativas. De acuerdo con Souza et al. (2012), para seleccionar un método de evaluación ambiental y de toma de decisiones debe llevarse en consideración su adaptabilidad, o sea la evaluación de pocas/varias alternativas, la utilización de pocos/varios criterios de evaluación, el uso de análisis cualitativa o cuantitativa, la primacía según criterios o pesos cualitativos y el uso de formas de evaluación asociativas, o no, con el objeto de comparar las alternativas. La cuestión de base consiste en la unidad de medida a ser utilizada para dimensionar aspectos tan diversos como los ambientales, por ejemplo, la contaminación del aire, los efectos sobre la salud o los impactos sobre una determinada estructura social y cultural. En líneas generales, el primer grupo de técnicas y métodos busca una cantidad de estos aspectos, evaluándolos en términos monetarios. El segundo, partiendo del presupuesto del difícil establecimiento de una unidad de medida común, busca aplicar escalas valorativas a los diferentes impactos medidos, originalmente, en sus respectivas unidades físicas o en términos cualitativos. De acuerdo con los estudios analíticos de metodología de evaluación de impactos ambientales, propuestos nacional e internacionalmente, es de fundamental importancia la incorporación de un conjunto de criterios básicos en los actuales métodos de análisis. Las metodologías han de ser flexibles, aplicables en cualquier fase del proceso de planificación y desarrollo y han de revisarse constantemente, en función de los resultados obtenidos y de la experiencia adquirida. Deben ser adecuadas para poder efectuar un análisis integrado, global, sistemático e interdisciplinario del medio ambiente y desus muchos componentes. Algunos criterios pueden ser la agregación de los aspectos físicos, biológicos y socioeconómicos; la inclusión del factor tiempo, la utilización de indicadores que faciliten la tarea de prospección y sectorización del área o la utilización de un mecanismo que permita sumar los impactos parciales para obtener el impacto total sobre el lugar; con la capacidad de extrapolar y archivar datos para la aplicación en otras áreas a ser estudiadas y aplicación en diferentes escalas y, además, que permitan la participación pública en la toma de decisiones. Todos esos criterios intentan hacer cada vez más eficaces las evaluaciones y la interpretación del ambiente, 
permitiendo el consecuente análisis de viabilidad e identificación de posibles alternativas para la prevención, recuperación y/o reconstitución ambiental. Como ya fue mencionado anteriormente, no existe una metodología completa e ideal que atienda a los diferentes estudios de impacto ambiental existentes y sus respectivas fases. La selección de la (s) más apropiada (s), además de atender los requisitos y normas legales establecidos para la ejecución delos estudios, es función del tiempo, de los recursos financieros disponibles y, en algunos casos, de los datos existentes. Sin embargo, es importante seleccionar metodologías en la medida en que sus principios puedan ser utilizados os adaptados a las condiciones específicas de cada estudio ambiental y de cada realidad local y nacional. Básicamente, existen las siguientes líneas metodológicas desarrolladas para la evaluación de impactos ambientales: Metodologías Espontáneas ('Ad hoc'); Listas de Control ('Check-List'); Matrices de Interacciones; Redes de Interacciones ('Networks'); Metodologías de Cantidad; Modelos de Simulación; Mapas de Superposición ('Overlays'); Proyección de Escenarios, otras. A continuación presentamos las metodologías concretas que se siguen enlas evaluaciones de impacto, o sea, los métodos de Identificación, de Predicción y de Interpretación.

\section{REFERENCIAS}

ANDRADE, G. F.; SANCHEZ, G. F.; ALMEIDA, J. R. Monitoramento e avaliação em projetos de recuperação de áreas degradadas. Revista Internacional de Ciências, v.4, p.13-26, 2014. DOI: http://doi.org/10.12957/ric.2014.13833

ALMEIDA, J. R.; SILVA, C. E.; SILVA, C. V. V.; AGUIAR, L. A.; GARCIA, V. S.; SOUZA, C. P.; LENZ, E. R. S.; LINS, G. A.; ALMEIDA, S. M.. Multifatorialidade em saúde ambiental. Environmental Scientiae, v.1, n.2, p.26-47, 2019. DOI: http://doi.org/10.6008/CBPC2674-6492.2019.002.0002

ALMEIDA, J. R.; SILVA, C. E.; SILVA, C. V. V.; AGUIAR, L. A.; GARCIA, V. S.; SOUZA, C. P.; LENZ, E. R. S.; LINS, G. A.; ALMEIDA, S. M.. Política e economia de vigilância em saúde ambiental. Environmental Scientiae, n.2, p.1-25, 2019. DOI: http://doi.org/10.6008/CBPC2674-6492.2019.002.0001

ALMEIDA, J. R.; SILVA, C. E.. Análisis "ex-post-facto" del estado consumatório denotativo de los principios de la declaración del rio en padrón personal. Educationis, Aquidabã, v.1, n.1, p.17-21, 2013. DOI: http://dx.doi.org/10.6008/ESS2318 - 3047.2013.001.0002
SOUZA, F. M. N.; SILVA, C. E.; AGUIAR, L. A.; ALMEIDA, J. R.. Análise de riscos como instrumento para sistemas de gestão ambiental. Revista Ibero-Americana de Ciências Ambientais, Aquidabã, v.3, n.1, p.17-41, 2012. DOI: https://doi.org/10.6008/ESS2179-6858.2012.001.0002

SOUZA, F. M. N.; SILVA, C. E.; AGUIAR, L. A.; ALMEIDA, J. R.. Proposta para utilização da simulação computacional em análise de risco, avaliação de desempenho e sistemas de gestão ambiental. Revista Ibero-Americana de Ciências Ambientais, v.2, n.2, p.39-63, 2011. DOI: https://doi.org/10.6008/ESS2179-6858.2011.002.0003

LINS, G. A.; BEZERRA, L. G. E.; MOTA, M. J. P.; ROCHABARBOSA, O.; ALMEIDA, J. R.. A ecologia de estrada sob a ótica do licenciamento ambiental. Sustinere: Revista de Saúde e Educação, v.3, p.153-160, 2015. DOI: http://dx.doi.org/10.12957/sustinere.2015.20143

A CBPC - Companhia Brasileira de Produção Científica (CNPJ: 11.221.422/0001-03) detém os direitos materiais desta publicação. Os direitos referem-se à publicação do trabalho em qualquer parte do mundo, incluindo os direitos às renovações, expansões e disseminações da contribuição, bem como outros direitos subsidiários. Todos os trabalhos publicados eletronicamente poderão posteriormente ser publicados em coletâneas impressas sob coordenação da Sustenere Publishing, da Companhia Brasileira de Produção Científica e seus parceiros autorizados. Os (as) autores (as) preservam os direitos autorais, mas não têm permissão para a publicação da contribuição em outro meio, impresso ou digital, em português ou em tradução. 